\title{
Median root prior and ordered subsets in Bayesian image reconstruction of single photon emission tomography.
}

Alain Seret.

Service des radioisotopes, Hopital Erasme (ULB), Brussels, Belgium.

Correspondance author: Alain Seret, Service des radioisotopes, Hopital Erasme (ULB), route de Lennik 808, B-1070 Brussels, Belgium.

fax: $32-2-5556800$

e-mail:aschout@resulb.ulb.ac.be 
Post-print author

Abstract

Median root prior allows Bayesian image reconstruction without any a-priori knowledge of the final solution. It limits the noise generated by maximum likelihood-expectation maximization including when the ordered subsets accelerating procedure is used. Therefore the number of iterations can be optimized to get the best resolution for cold lesions. Moreover the higher the number of subsets, the better the contrast with optimal results for subsets containing between 4 to 8 projections.

Key words: Emission tomography - Median root prior - Ordered subsets Iterative Bayesian reconstruction - Maximum likelihood-expectation maximization. 
Post-print author

INTRODUCTION

Recently, an attractive Bayesian method has been proposed in order to reduce noise in maximum likelihood-expectation maximization (MLEM) iterative reconstruction of positron emission tomography (PET) [1]. The idea of Alenius and Ruotsalainen is quite simple. The MLEM scheme is applied as usual. And at the end of each iteration, a penalty based on the Bayesian rule is applied to the reconstructed data. The penalty is weighted by the Bayesian parameter and is implemented as a one step late local median root prior (MRP). The local median root prior considers that the noise is responsible for any variation located on one pixel and tends to eliminate it. The penalty stems for an a-priori consideration that reconstructed images should be smooth. It is applied on the images from the previous MLEM iteration.

MRP generates quantitatively stable solutions with respect to the number of iterations [1]. This is a very interesting property since it is well known that the increase in the iteration number tends to generate less quantitatively accurate solutions in the MLEM process [1-3]. Among the major advantage of MRP, two are worth mentionning. In contrast to the smoothing methods, the median root prior does not alter the root signal. No a-priori knowledge of the final solution is needed in opposition to other Bayesian methods.

MRP is also attractive for MLEM reconstruction of single photon emission tomography (SPECT). Indeed, SPECT encounters the same noise and quantitative problems as PET. For application in clinical routine, the use of an accelerating procedure is highly desirable. This is for example particularly true in low count density regions where a large number of iterations is required [3]. An accelerating procedure that would limit the noise generated by the numerous iterations would therefore be very appreciable. Alenius [1] claims

Published in European Journal of Nuclear Medicine 1998;25:215-219 
Post-print author

that MRP is compatible with the ordered subsets (OS) accelerating procedure [4]. However he mentions that the results should be dependent on the way the subsets are chosen. Our main purpose is to analyse more deeply the compatibility between OS and MRP.

One major interest of MLEM and MLEM+OS is the positivity of the solution $[3,5,6]$. Therefore, the observation of cold regions is unequivocal. This contrasts to filtered backprojection (FBP) where negative values and therefore artefactual cold regions are generated by the filtering process [7]. However, MRP leads to a worse reconstructed resolution than MLEM [1]. Moreover, it has been demonstrated that contrast recovery for cold rods is more dependent on SPECT resolution than it is for hot rods [8]. MRP was therefore tested on a SPECT acquisition of a cylindrical phantom with cold rods of various diameters. 
For comparative purpose with the work of Alenius [1], the same Shepp-Logan phantom was generated by the computer.

The cold rods phantom was a perpex cylinder of $19 \mathrm{~cm}$ inner diameter and $13 \mathrm{~cm}$ height. It was filled with a radioactive aqueous solution of Tc99m-pertechnetate. The largest cold rod (30 mm diametrer) was located on the cylinder axis, the six others $(25,20,15,10,7$ and $5 \mathrm{~mm}$ diameter) were located at $7 \mathrm{~cm}$ from the axis.

The tomographic acquisition was performed with a Sophy DSX (SMV, Buc, France) camera. 64 projections in $128 * 128$ format were recorded using a freely defined and closed $\left(360^{\circ}\right)$ contour for a total of 4 Mcounts in the $141 \mathrm{keV} / 24 \%$ window. These data were also used in the compressed $64{ }^{\star} 64$ format.

The MLEM+OS software was written in Vision Programming Language (the SMV adaptation of PV-WAVE to nuclear medicine) and called a projector/ backprojector that was written in C. In this way, modifications of the code such as the implementation of the one step late median root prior were easily and rapidly performed. No pre- or post-reconstruction filter was applied. No attempt was made to correct for attenuation, diffusion or resolution variation with the distance.

The median root prior was calculated as described in the original paper of Alenius and Ruotsalainen [1]. If $\lambda_{n}(i)$ stems for the set of reconstructed data after the $n$-th iteration and $i$ is the pixel index, MRP is applied in the following way: 
Post-print author

where subscript MLEM (MRP) means after completion of the MLEM (MRP) process. $\operatorname{Med}(\lambda, i)$ is the median over a neighbouroud at pixel i ( $3 \times 3$ pixels was used) and $\beta$ is the Bayesian parameter. $\beta$ value should be kept below unity to avoid risk of overcorrection (Eq. 1). For $\beta$ set at 0 , no penalty is applied and the method becomes the standard MLEM method. At the n-th iteration, the penalty factor is computed from the result of the previous (n-1)-th MLEM +MRP iteration (one step late). More details about the one step late algorithm and Bayesian reconstruction methods can be found in references 1 and 9. All subsets were first all submited to the maximization as conventionally done in MLEM+OS. Thereafter, the penalty (Eq. 1) was applied to the whole set of reconstructed data and a new iteration was started until the preset number of iterations was reached. A second implementation was used, whereby each subset was submitted to the penalty after its maximisation. When all subsets were processed, a new iteration was started and the process was repeated until the preset number of iterations was reached. In this last configuration, the penalty was applied a number of times equal to the product of the subset number by the iteration number.

For each reconstruction, two profiles were extracted from the mid transverse slice: one through the 20 and $25 \mathrm{~mm}$ cold rods and one through the 5, 30 (central rod) and $15 \mathrm{~mm}$ cold rods. Contrast for the largest cold rods (15 to $30 \mathrm{~mm}$ ) was computed as

$$
C=1-\text { Arod } / A B G
$$

Arod was the activity in a circular ROI of 13 pixels. ABG was the activity in an annular ROI. Both ROls were centered on the rod axis. The smaller rods $(5,7$, $10 \mathrm{~mm}$ ) were not sufficiently resolved to allow contrast computation. 
Post-print author

RESULTS

For the Shepp-Logan phantom, Fig. 1 illustrates the dependence of the value of an arbitrary chosen pixel and of the standard deviation in a large $\mathrm{ROI}$ on the number of iterations in the case of MLEM and MLEM+OS with and without MRP.

The noise reduction afforded by MRP is visually illustrated in Figs. 2 to 5 for MLEM and MLEM+OS. Figs. 4 and 5 further illustrate also the influence of the number of iterations on the reconstructed slices for MLEM+OS and MLEM+OS+MRP.

The profiles through the cold regions are illustrated in Fig. 3a for reconstruction conditions where the product iteration number by subset number is kept constant. The number of subsets does not influence the contrast without MRP (Fig. 3b). However with MRP, the larger the subset number the better the contrast (Fig. 3).

For 8 subsets and without MRP, the noise (Fig. 5a) and the contrast (Fig. 6) increase with the number of iterations. With MRP, the noise (Fig. 5b) is fairly constant and the contrast (Fig. 6) improves with the number of iterations.

The profiles through the other cold rods, the contrast for these other rods, the use of the $64{ }^{\star} 64$ format, the use of an odd number of subsets (i.e. non equal number of projections per subset), and the use of values of the Bayesian parameter larger than $0.3(0.5,0.7,0.9)$ lead to the same qualitative observations.

All these data were obtained with the first implementation of MRP. The second implementation was used with a product iteration number by subset number equal to 48 . The obtained images were very similar and the profiles were so close to each other that it was not possible to display them

Published in European Journal of Nuclear Medicine 1998;25:215-219 
Post-print author

in a readable figure. These images and profiles are alike those obtained for 48 iterations of MLEM illustrated in Figs. 2 (top row, left) and 3a, respectively. 
Post-print author

DISCUSSION

The curves for MLEM and MLEM+MRP of Figure 1 are very similar to the curves obtained by Alenius [1] for the same Shepp-Logan phantom. This demonstrates that Alenius's MLEM algorithm and our MLEM+OS algorithm behave similarly with MRP. From the point of view of pixel value (Figure 1a) and noise (Figs. 1 b, 2 and 3), MRP is clearly efficient in stabilizing the solution generated by MLEM and MLEM+OS iterative reconstructions. This property of MRP was first demonstrated in the original paper of Alenius and Ruotsalainen for MLEM [1] and is now extented to MLEM+OS.

Let us now discuss the influence of the subset number when the product iteration number by subset number is identical (Figs. 2 and 3 ). Differences appear only when MRP is used (Fig. 3). The higher the number of subsets, the higher the contrast. This indicates that the less the penalty is applied the better is the contrast. This is further confirmed by the second implementation of MRP where the penalty is applied after each subset maximisation. In this case, the penalty is applied the same number of times regardless of the subset number choice. Whatever the subset number is, the profiles and the contrast are identical. Subsets containing 4 or 8 projections appears to give the best contrast (Fig. 3 b) so that it can be concluded that 4 to 8 projections per subset give the best contrast.

For MLEM+OS and a same number of subsets, more iterations lead to an increase in noise (Figs. 1 and 4) and contrast (Figs. 5 and 6). The use of MRP reduces noise significantly (Figs. 1 and 4) without altering significantly the contrast (Fig. 6).

The useful range of the Bayesian parameter is between 0.3 and 0.9 [1]. The presented results were obtained for a value of the Bayesian parameter equal to 0.3 . This value was shown to be the best compromise between Published in European Journal of Nuclear Medicine 1998;25:215-219 
Post-print author

substantial noise reduction and acceptable resolution [1]. Observations made with Bayesian parameter fixed at 0.3 are also valid for larger values $(0.5,0.7$, 0.9) of the Bayesian parameter except for the contrast which is gradually poorer. The increase in the value of the Bayesian parameter was indeed shown to decrease the resolution [1]. In this sense the Bayesian parameter plays a similar role towards the compromise noise/resolution as does the filter cut-off frequency in filtered backprojection.

Same benefits in terms of contrast and noise reduction from MRP could probably be obtained with standard MLEM if the penalty is applied after a block of a few iterations instead of after each iteration. Such a procedure would lead to the loss of the time benefit obtained by OS and is therefore not indicated in the clinical routine.

In conclusion, the combined used of MRP and OS with MLEM readily limits the noise generated by MLEM. Moreover, a careful choice of the number of subsets allows to reach identical or better contrast than in simple MLEM +MRP. Subsets containing between 4 to 8 projections appear to give the best results. The number of iterations should be chosen on the basis of counting statistics alone, low count density regions requiring more iterations [3]. Identical noise reduction and contrast improvement should render the MLEM +OS+MRP method even more attractive that MLEM+MRP for studies where large number of iterations are required, for example in low counts density regions. The value of the Bayesian parameter is a compromise between noise reduction and acceptable resolution. It should be however kept in mind that values of the Bayesian parameter higher than 0.3 lead to substantial loss of resolution while leaving the noise more or less constant [1]. 
Post-print author

AKNOWLEDGEMENTS

The MLEM-OS software (IDL version) has been kindly provided by Johan Nuyts from U.Z. Gasthuisberg, K.U.Leuven, Belgium. 
Post-print author

REFERENCES

1. Alenius S, Ruotsalainen U. Bayesian image reconstruction for emission tomography based on median root prior. Eur J Nucl Med 1997; 24:258-265.

2. Chornoboy, E S, Chen, C J, Miller M I, Miller T R, Snyder D L. An evaluation of maximum likelihood reconstruction for SPECT. IEEE Trans Med Imaging 1990; 9: 99-110.

3. Walrand $\mathrm{S} \mathrm{H}$, van Embt $L \mathrm{R}$, Pauwels $\mathrm{S}$. A non-negative fast multiplicative algorithm in 3D scatter compensated SPET reconstruction. Eur J Nucl Med 1996; 23: 1521-1526.

4. Hudson H M, Larkin R S. Accelerated image reconstruction using ordered subsets of projection data. IEEE Trans Med Imaging 1994; 13: 601-609.

5. Shepp L A, Vardi Y. Maximum likelihood reconstruction for emission tomography. IEEE Trans Med Imaging 1982; MI-1: 113-122.

6. Lange K, Carson R. EM reconstruction algorithms for emission and transmission tomography. J Comput Assist Tomogr 1984; 8: 306-316.

7. Forstrom L A, Dunn W L, O'Connor M K, Decklever T D, Hardyman T J, Howarth D M. Technical pitfalls in image acquisition, processing and display. Semin Nucl Med 1996 26: 278-294.

8. Kojima A, Matsumoto M, Takahashi M, Hirota Y, Yoshida H. Effect of spatial resolution on SPECT quantification values. J Nucl Med 1989; 30: 508-514.

9. Green P J. Bayesian reconstructions from emission tomography data using a modified EM algorithm. IEEE Trans Med Imaging 1990; 9: 84-93. 
Post-print author

FIGURES

Fig. 1. (a) Counts in an arbitrary chosen pixel and (b) standard deviation in a large ROI for reconstruction of the Shepp-Logan phantom by MLEM (. . .),

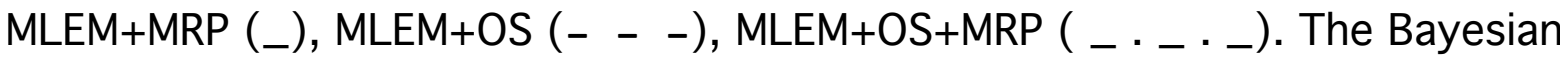
parameter in MRP was 0.3 and the subsets number 8 .

Fig. 2. Mid transverse slices obtained by iterative reconstruction in $128^{*} 128$ format. First column without MRP, second column with MRP (Bayesian parameter is 0.3 ). Iterative parameters were 1 subsets and 48 iterations for the first row, 4 subsets and 12 iterations for the second row, 16 subsets and 3 iterations for the third row. The lines indicate the image row used for the profiles of Fig. 3.

Fig. 3. (a) Profiles through the 5, 30 and $15 \mathrm{~mm}$ cold rods extracted from the slices of Fig. 2 for a number of iterations times the number of subsets equal to 48. Without MRP, all profiles are identical and shown in continuous line. With MRP, profiles are shown for $1(\ldots), 4\left(--_{-}\right)$and $16\left({ }_{-}\right.$. $\left._{-}\right)$) subsets. (b) Contrast of the 30 ( squares), 15 (diamonds) $\mathrm{mm}$ diameter rods for MLEM+OS (open symbols) and MLEM+OS+MRP (closed symbols) as a function of the number of subsets. The number of iterations is such that the product of these two parameters is equal to 48 . The Bayesian parameter is equal to 0.3 .

Fig. 4. Mid transverse slices obtained by iterative reconstruction in $128 * 128$ format. First column without MRP, second column with MRP (Bayesian parameter is 0.3 ). 8 subsets were used. Number of iterations was 2 for the Published in European Journal of Nuclear Medicine 1998;25:215-219 
Post-print author

first row, 6 for the second row, 12 for the third row. The lines indicate the image row used for the profiles of Fig. 5.

Fig. 5. Profiles through the 5, 30 and $15 \mathrm{~mm}$ cold rods extracted from the slices of Fig. 3 for 8 subsets and 2 (_), 6 (. . ) and 12 (- - ) iterations. (a) without MRP, (b) with MRP and the Bayesian parameter equal to 0.3.

Figure 6. Contrast of the 30 ( squares), 15 (diamonds) $\mathrm{mm}$ diameter rods for MLEM+OS (open symbols) and MLEM+OS+MRP (closed symbols) as a function of the number of iterations. The number of subsets is 8 and the Bayesian parameter is equal to 0.3 . 
Post-print author

Figure 1
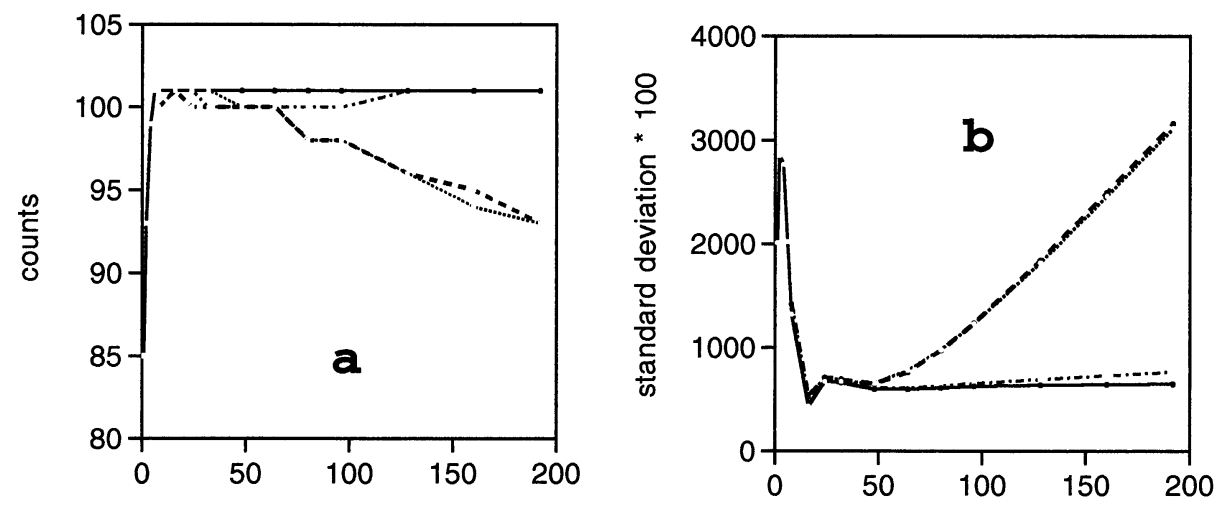

number of iterations * number of subsets

number of iterations * number of subsets

Figure 2

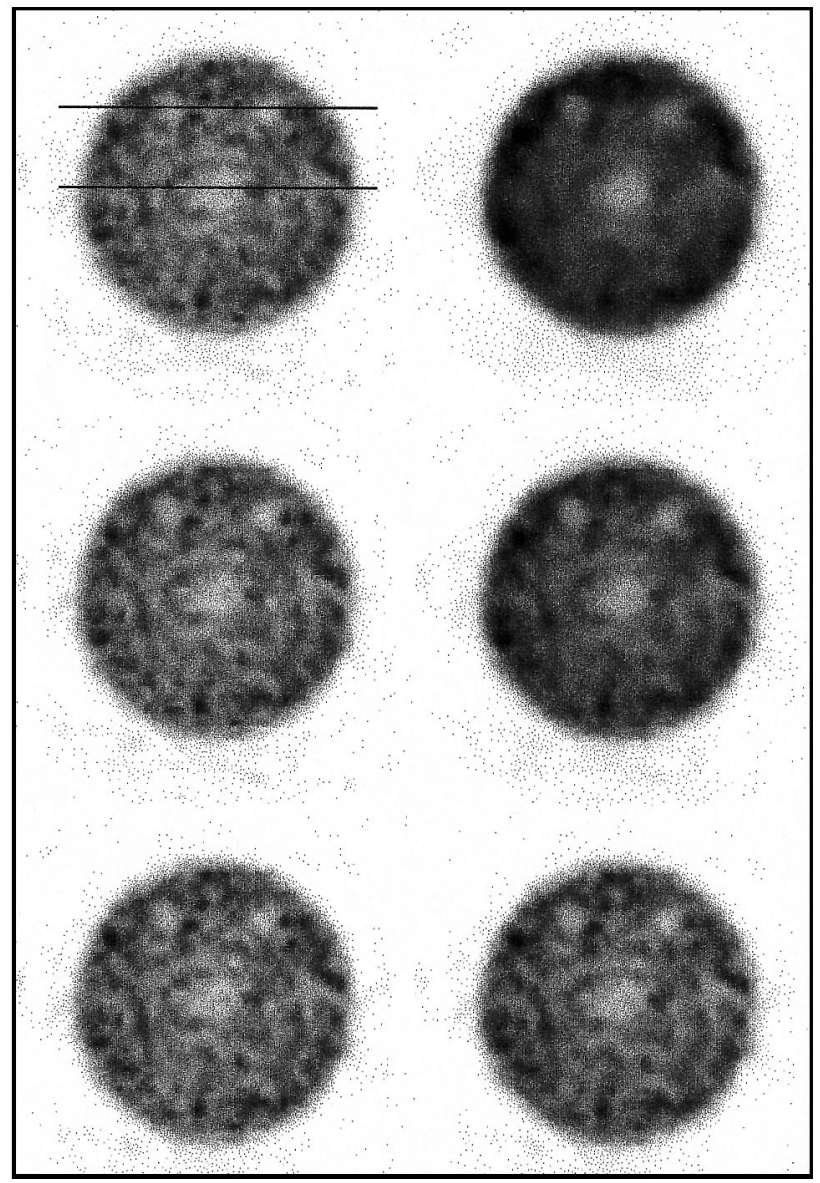

Published in European Journal of Nuclear Medicine 1998;25:215-219 
Post-print author

Figure 3
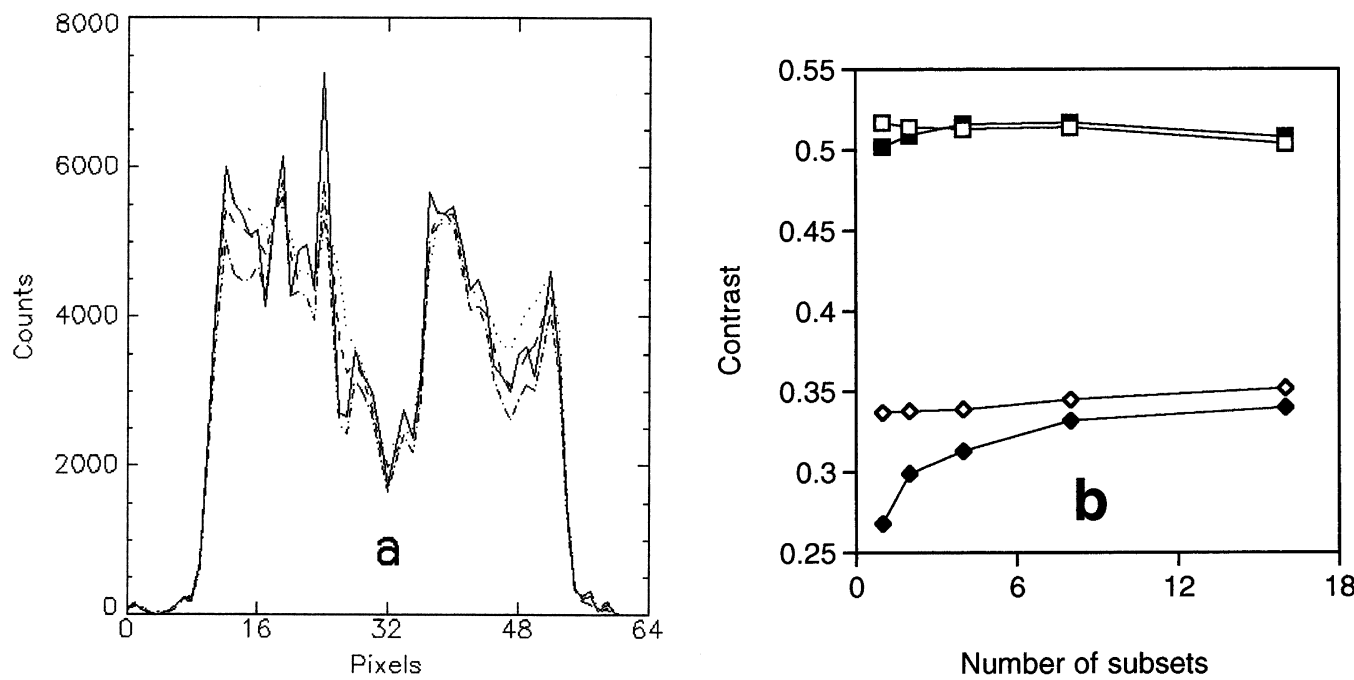

Figure 4

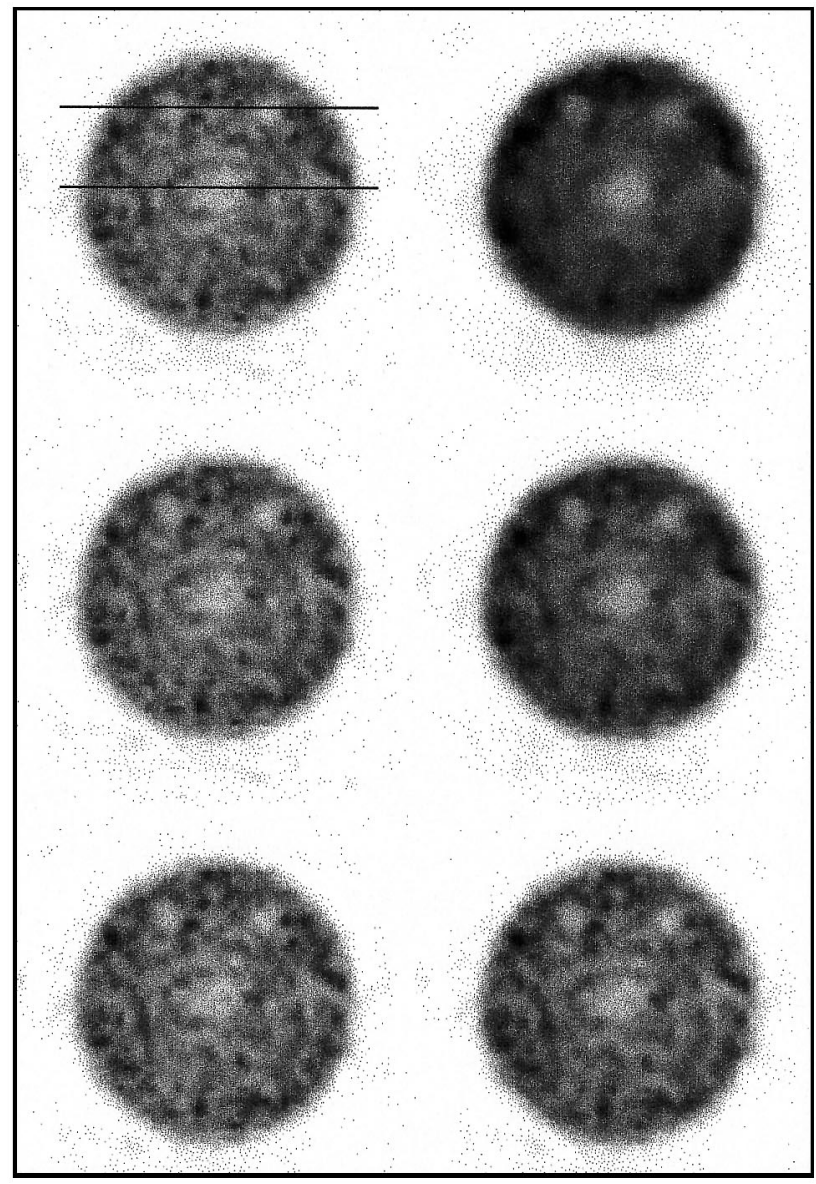

Published in European Journal of Nuclear Medicine 1998;25:215-219 
Post-print author

Figure 5
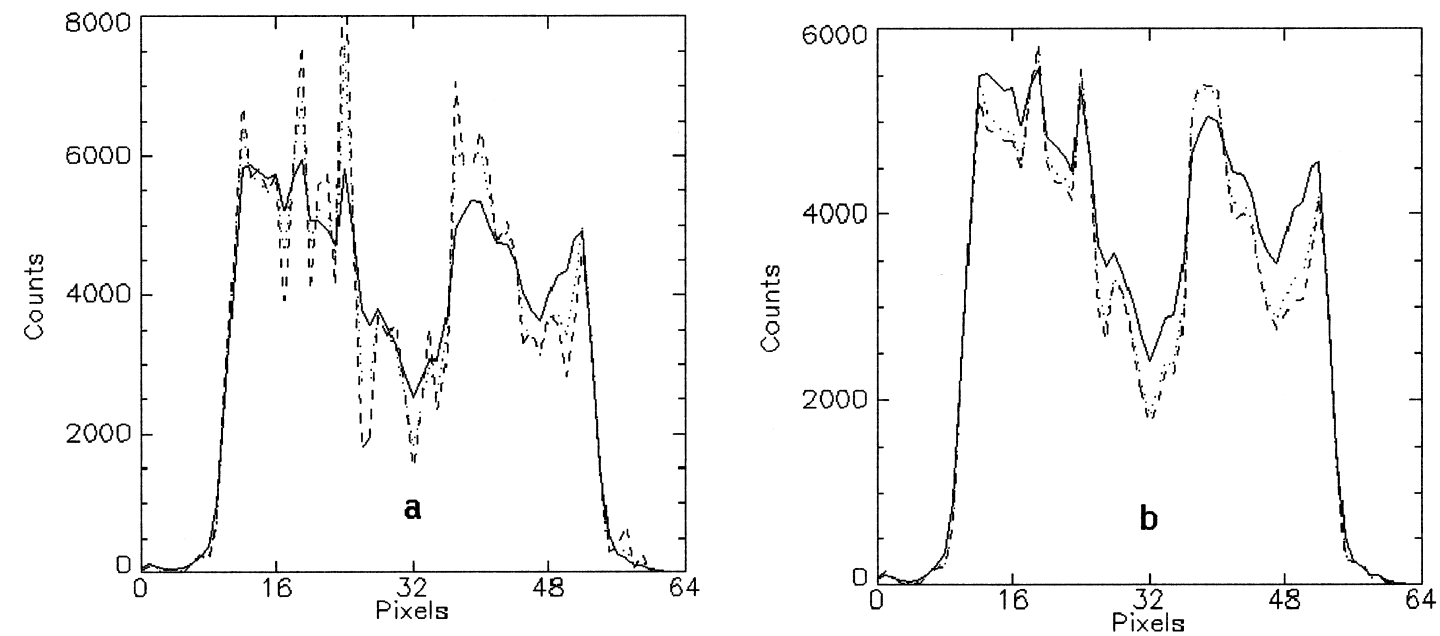

Figure 6

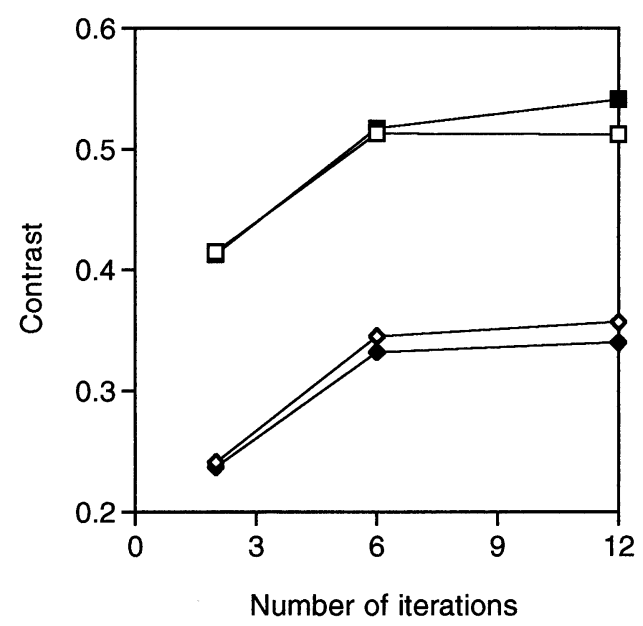

Published in European Journal of Nuclear Medicine 1998;25:215-219 